\title{
Le « Numeruswechsel » dans les témoins hébreux et grecs de Deutéronome 1
}

Par

\section{Hans Ausloos}

Université catholique de Louvain / F.R.S.-FNRS

$\mathrm{L}$ a critique textuelle biblique est un champ de recherches privilégié dans la carrière scientifique du professeur Jean-Claude Haelewyck. À cet égard, sa bibliographie extensive ne montre pas seulement un intérêt particulier pour l'évangile de Marc, mais aussi pour quelques livres vétérotestamentaires, comme Esther et Job. Comment donc honorer ce collègue de manière plus adéquate qu'avec une contribution sur la critique textuelle d'un livre biblique?

Sans doute, l'échange entre la $2^{\mathrm{e}}$ personne du singulier et la $2^{\mathrm{e}}$ personne du pluriel dans le discours d'adieu de Moïse, qu'il adresse aux Israélites, compte parmi les caractéristiques les plus énigmatiques du livre du Deutéronome. Dans la littérature scientifique, ce changement est désigné par le mot allemand « Numeruswechsel». Bien que les lecteurs bibliques aient depuis toujours remarqué cette particularité, aucune analyse systématique de ce problème n'a été faite jusqu'aux publications de Willy Staerk et de Carl Steuernagel à la fin du XIX ${ }^{\mathrm{e}}$ siècle $^{1}$.

Dans le contexte des recherches historico-critiques, ces deux chercheurs sont à l'origine d'un intérêt pour le « Numeruswechsel » en tant que critère qui pourrait être utile à la reconstruction de l'histoire de la composition du livre du Deutéronome. Cependant, tout le monde n'a pas accepté leur utilisation du «Numeruswechsel» à cette fin. Déjà quelques années après la publication de leurs études, l'application du « Numeruswechsel » comme critère historico-

\footnotetext{
${ }^{1}$ Staerk 1894 ; Steuernagel 1894. Pour un état de la question exhaustif, voir Begg 1978, p. 7-1094. Voir également BEGG 1979 et 1994.
} 
critique a été rejetée par exemple par Alfred Bertholet, qui considérait ce phénomène plutôt comme une figure de style ${ }^{2}$.

Depuis lors, les recherches de l'origine et de la fonction du «Numeruswechsel » ont été marquées par ces deux points de vue différents. Afin de défendre leurs hypothèses respectives, les deux parties font régulièrement appel aux témoins textuels anciens, en particulier à la Septante (LXX) qui, souvent, ne coïncide pas avec le Texte massorétique (TM) ${ }^{3}$. À ce jour cependant, il n'y a pas encore d'analyse complète et systématique de la manière dont la LXX traite ce phénomène qui caractérise le TM du Deutéronome ${ }^{4}$. En général, il est admis que les traducteurs de la LXX aplanissent cette « irrégularité » du texte dans certains passages, tout en conservant le « Numeruswechsel» dans d'autres ${ }^{5}$. Pour la plupart, les spécialistes conviennent qu'il n'y a pas de règle rendant compte de cette alternance par le traducteur de la LXX.

Dans ce contexte, je me focaliserai dans cette contribution ${ }^{6}$, que je dédie à Jean-Claude Haelewyck, sur la manière dont le traducteur grec du Deutéronome a traité le phénomène du «Numeruswechsel» dans le premier chapitre du livre ${ }^{7}$, en complément de mes analyses précédentes de Dt 12 et du phénomène particulier du «Numeruswechsel » entre la $1^{\mathrm{e}}$ et la $3^{\mathrm{e}}$ personne $^{8}$. En Dt 1, on rencontre ce phénomène dans les versets $8,17,20-21$ et 31.

\section{Dt 1,8}

Après quelques versets introductifs (Dt 1,1-2), on lit en Dt 1,3 que Moïse s'adresse aux Israélites (pluriel ; litt. : « fils d’Israël 》 - בני ישראל) , selon ce que YHWH lui avait ordonné à

\footnotetext{
${ }^{2}$ BERTHOLET 1899.

${ }^{3}$ Comme indiqué dans AusLoos 1999, les chercheurs qui utilisent le « Numeruswechsel » comme critère pour leur analyse historico-critique de la Bible hébraïque s'aventurent sur une pente glissante. Voir déjà l'avertissement de HEMPEL 1914, p. 8 : « (...) so sind die LXX-Lesungen doppelt genau auf ihre Sicherheit hin zu prüfen, ehe man sich ihnen anschließt. Es gilt dies um so mehr, als wir bei einer ganzen Reihe von Stellen beobachten können, daß LXX den Text absichtlich oder unabsichtlich geändert hat. Vor allem handelt es sich dabei um solche, wo nur für einen oder wenige Verse der (...) Numerus-Wechsel eintritt; hier war natürlich die Gefahr sehr groß, daß der Übersetzer in dem herrschenden Numerus ruhig weiter schrieb, ohne den Wechsel des M.T. zu beachten ».

${ }^{4}$ Voir, par exemple, WEVERS 1995 qui, bien qu'il fasse référence au style « deutéronomique », ne mentionne pas le problème du « Numeruswechsel » en tant que tel.

${ }^{5}$ Dans leur introduction à la traduction française de la LXX, Dogniez et Harl se contentent de traiter de manière très concise le problème du « Numeruswechsel » : «Par exemple, l'alternance dans le texte hébreu du singulier et du pluriel, 'tu' et 'vous', pour désigner le destinataire des discours de Moïse, est globalement respectée par le traducteur - alternance qui sert de critère aux historiens du texte hébreu pour distinguer les diverses sources du livre. Si l'on note cependant d'assez nombreuses différences, qui semblent dues à la volonté d'éviter les brusques changements de personnes, ce souci d'uniformiser le texte à l'intérieur d'un même passage ne se montre pas de façon systématique tout au long du livre » (DognIEZ, HARL 2007, p. 30). Voir également LoHFINK 1963, p. 3031 : « Die Tendenz von Sam und LXX ist, zu harmonisieren, sodass man im allgemeinen dem TM folgen kann ». Il me semble d'ailleurs trop simple de postuler que ces variantes n'ont aucune valeur pour la critique textuelle du livre de Deutéronome (VEIJOLA 1988, p. 250).

${ }^{6}$ Je remercie cordialement le Drs. Simon Naveau d'avoir corrigé le français de cette contribution.

${ }^{7}$ Les éditions suivantes ont été utilisées : MCCARThy 2007 pour le TM, WeVERS 2006 pour la LXX, TAL FLORENTIN 2010 pour le Pentateuque samaritain (SamP) et ULRICH 2010 pour les manuscrits bibliques de la Mer morte.

${ }^{8}$ Ausloos 2018.

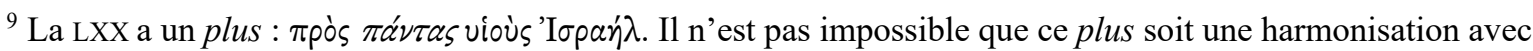

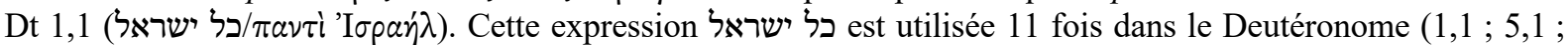

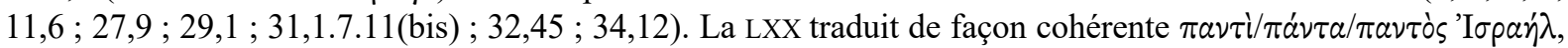


leur (pluriel : אלהם) sujet. Dès le début du livre, les Israélites sont donc désignés au pluriel,

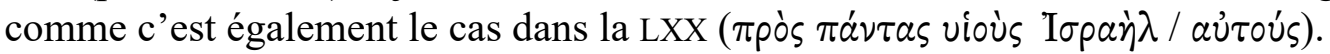

Lorsque Moïse entame son discours aux Israélites, il se considère comme l'un d'entre eux, et s'adresse donc à eux à la $1^{\text {re }}$ personne du pluriel. Ainsi, en Dt 1,6, Moïse fait référence à

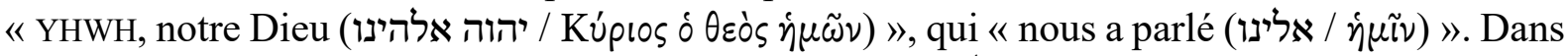
le discours qui suit, introduit par la forme hébraïque לאמר, Moïse cite les paroles de Dieu à l'adresse des Israélites. Comme Moïse, YHWH parle aux Israélites à la $2^{\mathrm{e}}$ personne du pluriel : «Vous avez longtemps demeuré (רב לכם שבת) dans cette montagne » (Dt 1,6), « Tournez-vous (פנו), reprenez votre route (וסעו לכם), (ובאו) (Dt 1,7). La LXX suit de près les formes

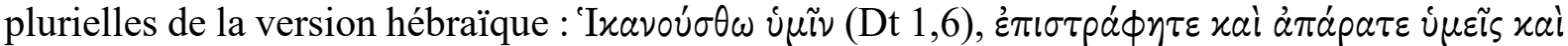

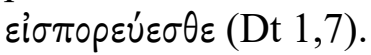

C'est en Dt 1,8, où YHWH s'adresse encore aux Israélites, qu'on rencontre le premier « Numeruswechsel » dans le texte hébreu du livre du Deutéronome (TM) ${ }^{10}$ : «Regarde (ראה) ». Cependant, cette forme à la $2^{\mathrm{e}}$ personne du singulier - qui, dans le TM, a sans aucun doute la fonction d'une simple interjection ${ }^{11}$ - est immédiatement suivie de phrases dans lesquelles YHWH s'adresse à nouveau aux Israélites à la $2^{\mathrm{e}}$ personne du pluriel : « J'ai mis le pays devant

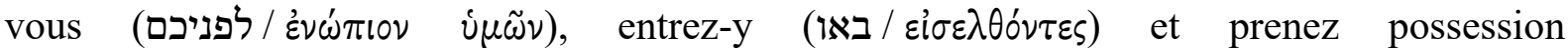

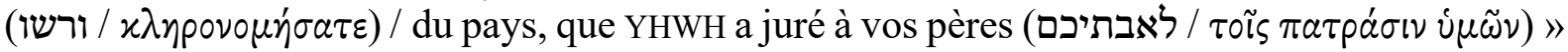
(Dt 1,8$)^{12}$.

Il est remarquable que la version de la LXX de Dt 1,8 ne suit pas le « Numeruswechsel » du TM. Ici, le traducteur utilise la forme plurielle ${ }^{\prime} \delta \varepsilon \tau \varepsilon^{13}$. Le SamP va dans la même direction : il lit également une forme plurielle (ראו) ; il en va de même pour le fragment qumrânien 2QDeut ${ }^{\mathrm{a}}$ (אוֹ et, en tant que simple exclamation, peut être traité comme indéclinable. Néanmoins, sa conclusion que les versions, qui lisent un équivalent au pluriel, ne signifie pas nécessairement qu'elles ont une forme au pluriel dans leurs Vorlagen respectives, est plus discutable ${ }^{15}$. Le fait que 2QDeut ${ }^{\mathrm{a}}$ - le témoin textuel le plus ancien de Dt 1,8 - lise également une forme plurielle, rend plausible une Vorlage différente ${ }^{16}$.

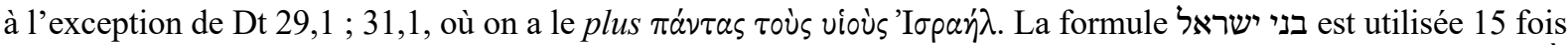
dans le Deutéronome : 1,$3 ; 3,18 ; 4,44.45 ; 28,69 ; 31,19$ (bis) ; 31,22.23;32,8.51(bis) ; 33,1;34,8(bis). À

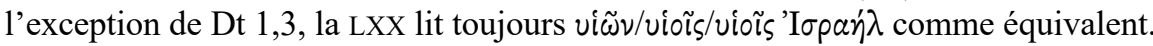

${ }^{10}$ Ce cas n'est pas mentionné par CAZELLES 1967, p. 208, qui considère Dt 1,21 comme le premier verset témoignant d'un « Numeruswechsel».

11 JOÜON, MURAOKA 1993, $§ 105$ : « An imperative can become pure interjection ».

12 Il faut remarquer que YHWH, par opposition à la première partie de Dt 1,8 où la $1^{\mathrm{e}}$ personne est utilisée, se réfère à lui-même à la $3^{\mathrm{e}}$ personne dans la $2^{\mathrm{e}}$ partie du verset.

${ }^{13}$ Comme en Dt 1,$21 ; 4,25$ : ‘’ $\delta \varepsilon \tau \varepsilon$. L'équivalent singulier idov́ est attesté en Dt 2,24.31;11,26;30,15, tandis

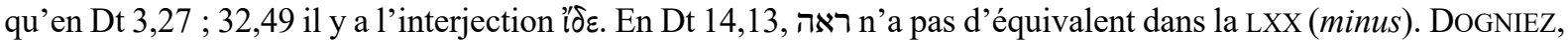
HARL 2007, p. 113 traduisent par « voyez »; cependant, elles ne prêtent pas attention à cette variante de la LXX. Déjà HEMPEL 1914, p. 8 considérait le pluriel de la LXX en Dt 1,8 comme une harmonisation par le traducteur de la LXX, comme 1'a fait KÖNIG 1917, p. 67.

${ }^{14}$ Voir également LABERGE 1985, p. 131-132, qui remarque que dans la Traduction cecuménique de la Bible le pluriel («Voyez ») est employé « sans doute à cause du contexte » (p. 131 n. 10).

${ }^{15}$ MCCARTHY 2007, p. 49*.

${ }^{16}$ Voir Den Hertog, LABAhn, Pola 2011, p. 537 : « Die breite Bezeugung spricht für eine eigenständige Texttradition ». 
Dans la suite du discours de YHWH en Dt 1,8, un autre « Numeruswechsel » se présente : selon le TM, YHWH commence à parler de lui-même à la $1^{\text {re }}$ personne du singulier (נתתי / $\pi \alpha a \delta \delta \varepsilon \delta \omega \varkappa \alpha)^{17}$, dans sa référence à son serment à Abraham, Isaac et Jacob ; mais il continue ensuite à la $3^{\mathrm{e}}$ personne. En tant que tel, YHWH se réfère à lui-même comme sujet explicite de la phrase (הארץ אשר נשבע יהוה). Cette référence à la promesse du pays qu'il a faite

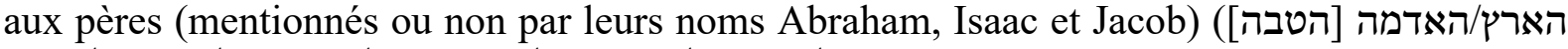
fait partie de la phraséologie typique du Deutéronome, où elle apparaît 19 fois. Dans douze cas, on réfère au serment de YHWH à la $3^{\mathrm{e}}$ personne (נשבע - Dt 1,8;6,10.18.23;7,13;8,1;11,9.21;26,3; $28,11 ; 30,20 ; 31,7)$; dans six autres cas, YHWH s'adresse aux Israélites à la $1^{\text {re }}$ personne (נשבעתי) - Dt 1,35;10,11;31,20.21.23;34,4). Ce n'est qu'en Dt 26,15, où Moïse s'adresse à YHWH, que le verbe est à la $2^{\mathrm{e}}$ personne (נשבעת).

En général, le texte de la LXX est en accord avec le $\mathrm{TM}^{18}$. On ne découvre une variante que

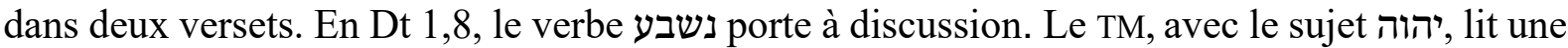
$3^{e}$ personne du singulier au niphal (את הארץ אשר נשבע יהוה לאבתיכם). La LXX en revanche, présente un minus pour le sujet יהוה et traduit le verbe par une $1^{\text {re }}$ personne du pluriel à l'indicatif

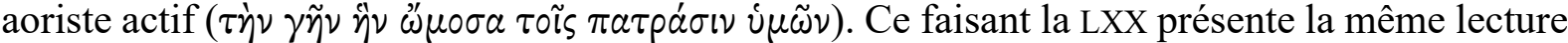
que le SamP (את הארץ אשר נשבעתי לאבתיכם). 4QDeuth , au contraire, suit le TM en Dt 1,8. 2QDeut ${ }^{\mathrm{a}}$ (הארץ אשר נשבע יהם לאבר) ne permet pas de tirer de conclusion ${ }^{19}$. En Dt 31,23, c'est le contraire qui se produit. Ici, une $1^{\text {re }}$ personne dans le TM a pour équivalent une $3^{\mathrm{e}}$ personne dans la LXX, avec le plus xúplos comme sujet.

En ce qui concerne Dt 1,8, il est difficile de retracer l'origine du « Numeruswechsel », à la fois dans le TM et dans la variante dans la LXX. Selon McCarthy, ce sont les LXX et le SamP qui ont harmonisé avec la majorité des cas où la formule est utilisée à la $3^{\mathrm{e}}$ personne ${ }^{20}$. Si sa suggestion est correcte - d'après elle, on pourrait surtout référer à Dt 1,35 qui fait partie du contexte immédiat de Dt 1,8, et qui lit נשבעתי / / $^{\prime} \mu \sigma \sigma \alpha-$-, on peut néanmoins se demander pourquoi cette harmonisation n'a pas été introduite dans les autres passages où la formule se trouve à la $1^{\text {re }}$ personne dans le TM. Peut-être, comme Wevers le suggère, la variation est-elle la conséquence d'une certaine négligence des copistes de la LXX, qui étaient sans doute « thoroughly familiar with these formulaic expression with the result that parallel passages have played havoc with the textual transmission $»^{21}$; d'ailleurs, toujours selon Wevers « the same kind of influence was also present with Hebrew copyists $»^{22}$. Selon Otto, le « Numeruswechsel » dans le TM est témoin des différentes couches rédactionnelles ${ }^{23}$, tandis que Rüterswörden le considère comme typique d'un style royal ${ }^{24}$.

Après le bref discours direct de YHWH en Dt 1,6-8, cité par Moïse, Moïse lui-même reprend son adresse en Dt 1,9. Comme Dieu l'a fait dans le discours cité, il s'adresse aux Israélites à la $2^{\mathrm{e}}$ personne du pluriel : «Je vous (אלכם / זpòs ú $\mu \tilde{a} \varsigma$ ) dis 》. De même, dans le discours direct qui suit, les Israélites sont systématiquement présentés au pluriel - la LXX s'aligne

\footnotetext{
${ }^{17}$ Quelques manuscrits $\left(\mathrm{B}, 82,630^{\mathrm{c}}\right)$ lisent $\pi \alpha$ a $a \delta \varepsilon ́ \delta \omega x \varepsilon \nu$. Voir WeVERs 2006, p. 56.

${ }^{18}$ WEVERS 1978, p. 86.

${ }^{19}$ Ausloos 2022, p. 51-57.

${ }^{20}$ MCCARTHY 2007, p. 50*.

${ }^{21}$ WeVERS 1978, p. 86.

22 WeVERS 1978, p. 86.

${ }^{23}$ Oтто 2012, p. 331.

${ }^{24}$ RÜTERSWÖRDEN 2006, p. 26.
} 
complètement sur le TM, bien qu'il y ait ici et là une petite variante - : "Je ne puis pas vous (אתכם / אלהיכם (

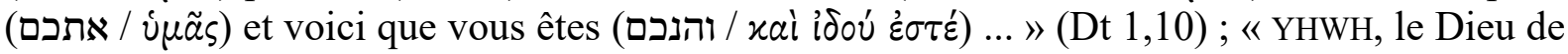

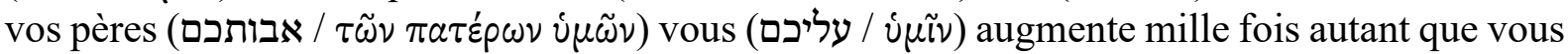

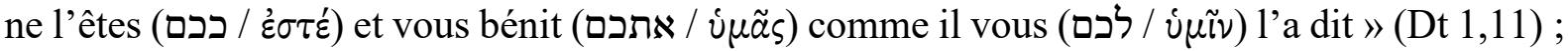

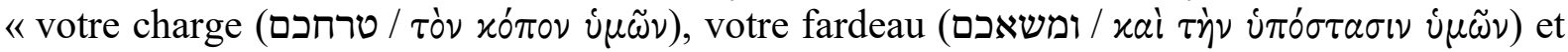

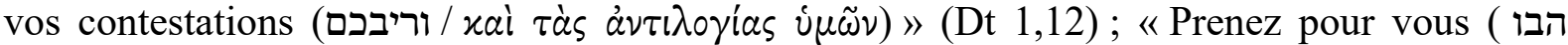

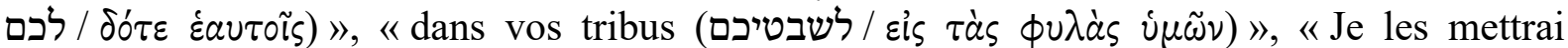

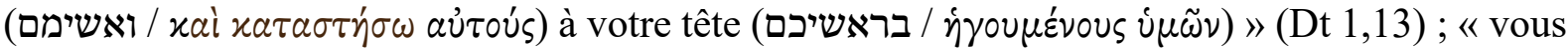
m'avez répondu (ותענו / xai à

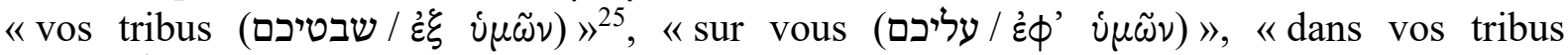

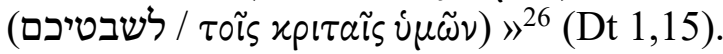

En Dt 1,16, Moïse rappelle à Israël, à qui il s'adresse à la $2^{\mathrm{e}}$ personne du pluriel, les ordres qu'il a donnés aux juges qu'on doit nommer («J'ai chargé vos juges [שפטיכם / שמא $\dot{u} \mu \tilde{\omega} \nu) »($ Dt 1,6). Dans un discours direct introduit par לאמר, Moïse ordonne ce que ces juges devront faire. Là où, en Dt 1,16, le TM lit un infinitif absolu (שמע בין אחיכם), la LXX présente

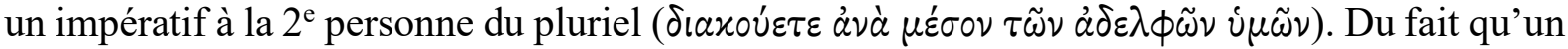
infinitif absolu isolé en hébreu peut avoir la fonction d'un impératif, cet équivalent grec rend correctement l'intention du texte hébreu - en tenant compte des versets alentour qui désignent les Israélites au pluriel -, comme c'est le cas dans la traduction de שפטתם (« vous jugerez 》 -

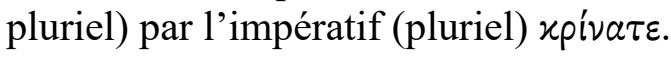

\section{Dt 1,17}

En Dt 1,17, on trouve un « Numeruswechsel » assez particulier. Alors que, dans le TM, Moïse continue à s'adresser aux juges futurs au pluriel (לא תגורו - תשמעון - לא תכירו), la LXX lit trois

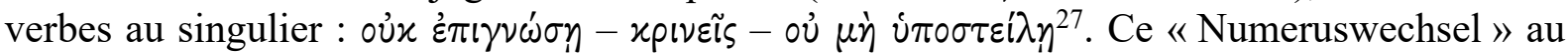
niveau de la LXX, qui n'est pas attesté dans le TM, est difficilement explicable. Peut-être le traducteur (ou un copiste ?) a-t-il été inspiré par les formes au singulier en Dt 16,19 לא תטה (לא תקח - לא תכיר, un verset qui, comme Dt 1,17, traite de l'impartialité des juges israélites ${ }^{28}$.

Malheureusement, il n'y a pas de texte hébreu existant présentant les verbes de Dt 1,17 au singulier ${ }^{29}$. Selon Lohfink, étant la lectio difficilior, la variante de la LXX pour ces trois verbes doit être considérée comme plus originale : ce serait un vestige d'une ancienne formule utilisée dans le contexte des nominations des juges («Richtereinsetzungsformel »), que l'auteur du Deutéronome vient de $\operatorname{citer}^{30}$. Quoi qu'il en soit, à partir de la $2^{\mathrm{e}}$ partie du v. 17, la LXX correspond de nouveau aux formes du pluriel du TM - quand un cas est trop difficile pour les

\footnotetext{
${ }^{25}$ La LXX a un minus en Dt 1,15. Là, où selon le TM, « Moïse a pris les chefs de vos tribus », la LXX lit «j'ai pris de vous des hommes ».

${ }^{26}$ Selon WEVERS 1995, p. 10, la LXX reflète une amélioration secondaire du texte sur l'arrière-fond de la caractérisation des officiers comme juges en Dt 1,16.

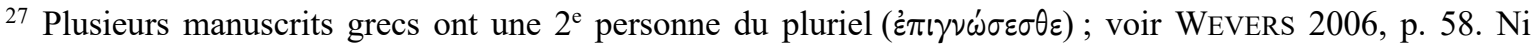
Doignez, Harl 2007, p. 115, ni Den Hertog, Labahn, Pola 2011, p. 538, ne vont plus loin que de mentionner la variante.

${ }^{28}$ La LXX de Dt 16,19 a des formes au pluriel. Dans la Biblia Hebraica Stuttgartensia (1972), comme dans l'édition précédente, la Biblia Hebraica (1935), J. Hempel suggère de supprimer Dt 1,17a (לא תכירו ... לאלהים הוא) à cause du singulier de la LXX : «f(o)rt(asse) $\mathrm{d}(\mathrm{e}) 1($ endum $)(\mathfrak{5}$ sing) ».

${ }^{29}$ En lisant לא תכירו (les autres verbes ne sont plus préservés), 4QDeut ${ }^{\mathrm{h}}$ suit le TM. SamP également suit le TM.

${ }^{30}$ LOHFINK 2004, p. 91.
} 


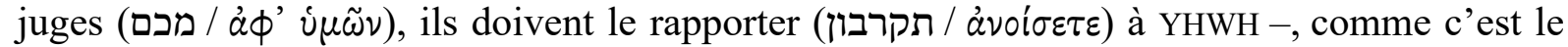

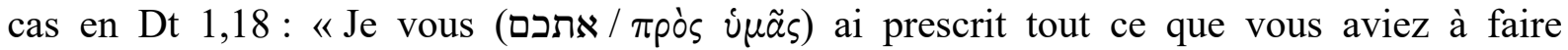

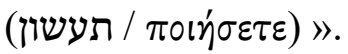

\section{Dt 1,20-21}

À partir de Dt 1,19, Moïse s'adresse de nouveau aux Israélites à la $1^{\text {re }}$ personne du pluriel, comme il l'a fait en Dt 1,6: « Nous sommes partis et nous avons parcouru depuis l'Horeb (מנסע

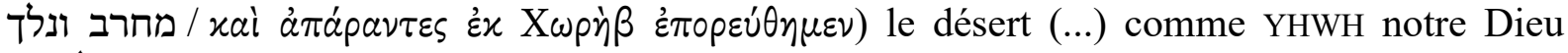

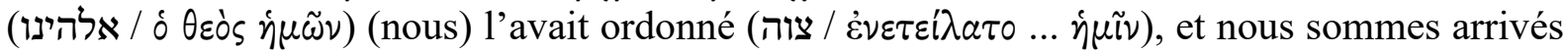

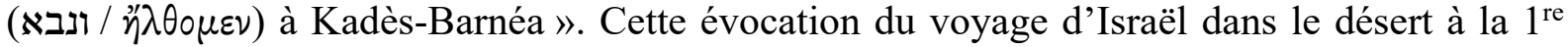
personne du pluriel est interrompue par un seul verbe à la $2^{\mathrm{e}}$ personne du pluriel : « le grand et

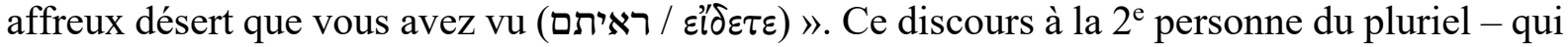
donne l'impression que Moïse se distancie des Israélites - se poursuit en Dt 1,20 : «Je vous

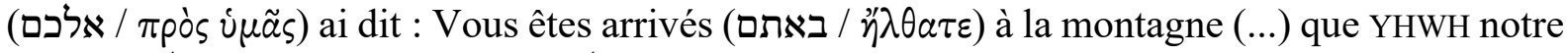

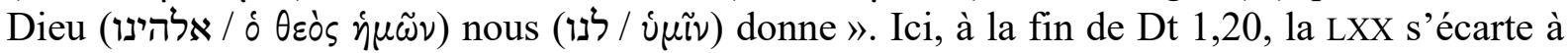
nouveau du TM (et du SamP). Au lieu de lire une $1^{\text {re }}$ personne du pluriel ( $\left.\dot{\eta} \mu \tilde{\omega} \nu\right)$ comme le TM, la LXX a une lecture à la $2^{\mathrm{e}}$ personne du pluriel $\tilde{u} \mu \tilde{\iota}^{31}$. Cette leçon à la $2^{\mathrm{e}}$ personne du pluriel pourrait être une harmonisation avec le contexte immédiat de Dt 1,20, où les Israélites sont mentionnés à la $2^{\mathrm{e}}$ personne du plurie $\mathrm{l}^{32}$. Néanmoins, selon Wevers, ú $\mu \tilde{\imath}$ pourrait être la leçon originale, car elle s'inscrit dans les formes de la $2^{\mathrm{e}}$ personne du pluriel de Dt 1,21 , alors que le TM a des formes de la $2^{\mathrm{e}}$ personne du singulier ${ }^{33}$.

Dans la suite de son discours en Dt 1,21, Moïse s'adresse aux Israélites au singulier, comme en Dt 1,8 : ראה. Dans la LXX, comme en Dt 1,8, le traducteur utilise la forme plurielle " $\delta \varepsilon \tau \varepsilon$. Mais, contrairement au Dt 1,8, en Dt 1,21, dans le TM, Moïse continue son discours en s'adressant aux Israélites au singulier ${ }^{34}$. La LXX, par contre, utilise systématiquement le pluriel, comme c'était le cas en Dt 1,8-16: "YHWH, ton Dieu te donnera (נתן יהוה אלהיך)

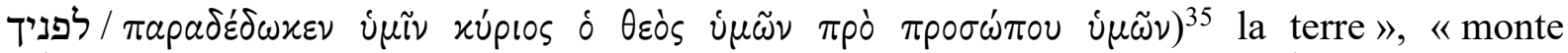

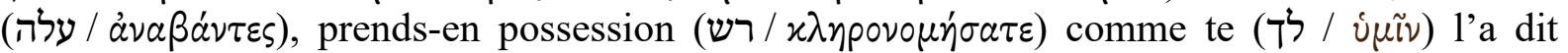

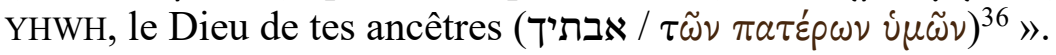

Dans la suite du v. 21, le TM continue au singulier (אל תירא ואל תחת), tandis que la LXX maintient le pluriel ( $\mu \grave{\eta} \phi \circ \beta \varepsilon \tilde{\sigma} \sigma \varepsilon \varepsilon \mu \eta \delta \varepsilon \dot{\varepsilon} \delta \varepsilon ı \lambda \iota \alpha ́ \sigma \eta \tau \varepsilon$ ). Ici, il n'est pas inimaginable que le traducteur de la LXX (ou sa Vorlage) ait envisagé d'harmoniser avec le verset suivant (v. 22) 37 . En effet, à partir de Dt 1,22, où c'est de nouveau Moïse qui parle lui-même directement aux

\footnotetext{
${ }^{31}$ Certains manuscrits grecs, dont le A, lisent $\dot{\eta} \mu \tilde{\nu}$, suivant ainsi le TM. Selon Wevers 1995, p. 12, cette lecture pourrait être à la fois un iotacisme ou une variante originale. Au lieu de $\eta^{\prime} \lambda \theta \alpha \tau \varepsilon, 630^{*}$ et 470 ont $\eta \lambda \theta 0 \mu \varepsilon \nu$.

32 Voir Den Hertog, Labahn, Pola 2011, p. 538 : « Angleichung an die Adressatengruppe des Volkes ».

${ }^{33}$ WEVERS 1995, p. 12 : «I would with some hesitation defend $\tilde{u} \mu \tilde{\nu}$ as original here ».

${ }^{34}$ Voir l'apparat critique par Hempel dans la Biblia Hebraica (repris dans la Biblia Hebraica Stuttgartensia) concernant Dt 1,21 : «f(o)rt(asse) tot(us) v(ersus) d(e)l(endum), quamvis plur(es) ». Selon CAZELLES 1967, p. 209, ce « Numeruswechsel» n'est probablement pas « a fantasy of the speaker ».

${ }^{35}$ La LXX a un plus : ن́ $\mu \tilde{\imath}$.

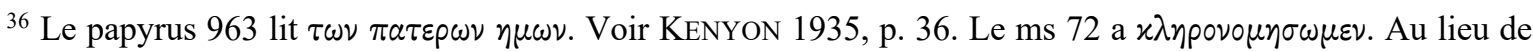
ú $\mu \tilde{\nu}$, plusieurs manuscrits ont $\eta \mu \omega v$.

37 Selon STAERK 1894, p. 58, le pluriel est original, tandis que STEUERNAGEL 1898, p. 5, considère le pluriel de la LXX comme une harmonisation secondaire avec le contexte, comme l'ont fait PUUKO 1910, p. 107 n.1, HeMPEl 1914, p. 8, KÖNIG 1917, p. 68, SMith 1918, p. 15, HoSPERS 1947, p. 14, Dogniez, HaRl 2007, p. 116, Отто 2012, p. 371.
} 
Israélites, le TM reprend la $2^{\mathrm{e}}$ personne du pluriel (la LXX fait de même) : « Vous vous êtes tous

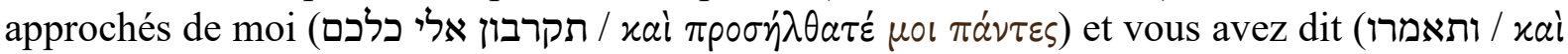
$\varepsilon l \pi \alpha \tau \varepsilon)$... ».

Dans le discours direct qui suit, dans lequel Moïse cite les Israélites (v. 22), le TM et la LXX

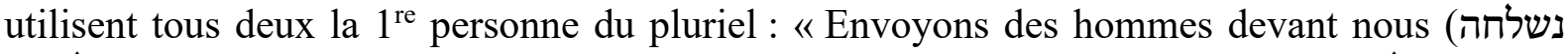
( אנשים לפנינו

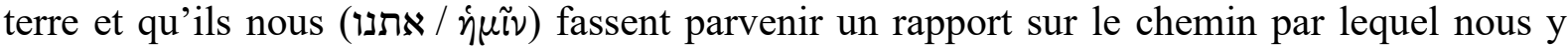

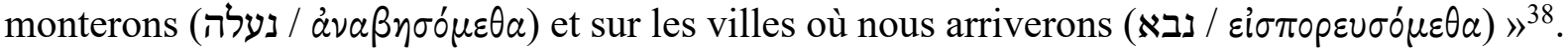

À partir de Dt 1,23, Moïse reprend la parole, en s'adressant de nouveau directement aux Israélites, à la $2^{\mathrm{e}}$ personne du pluriel (dans le TM comme dans la LXX : « J'ai pris douze hommes parmi vous [מכם / fait, en utilisant par conséquent la $3^{\mathrm{e}}$ personne du pluriel en Dt 1,24-25 (ils sont partis et ils ont

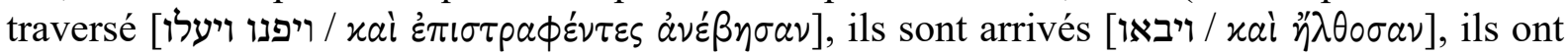

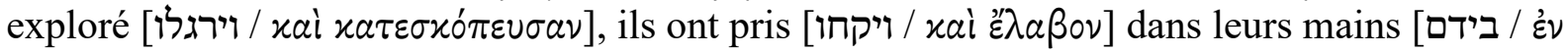

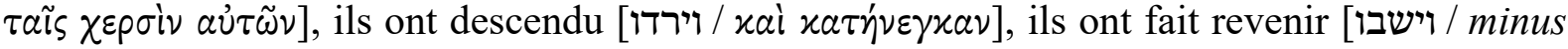

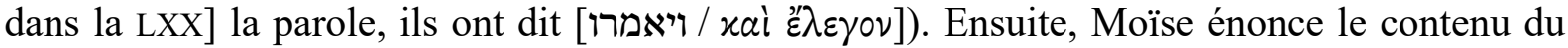
rapport des messagers à la $1^{\text {re }}$ personne du pluriel (Dt 1,25) - discours direct.

Ce faisant, il s'inclut comme un Israélite parmi son peuple (ils nous ont présenté [אלינו / הpòs $\dot{\eta} \mu \tilde{a} s]$, ils nous [אתנו / minus dans la LXX] ont fait un rapport, le pays que YHWH notre Dieu [וישבו אתנו OEòs dévie pas du TM.

\section{Dt 1,31}

En Dt 1,26, en s'adressant de nouveau directement aux Israélites, Moïse reprend la $2^{\mathrm{e}}$ personne du pluriel du v. 23, ce qui continue sans exception jusqu'au v. 30, dans le TM comme dans la LXX ${ }^{39}$ : «Vous n'avez pas voulu (לא אביתם / oủx $\left.\dot{\eta} \theta \varepsilon \lambda \eta \dot{\eta} \sigma \alpha \tau \varepsilon\right)$, vous vous êtes révoltés

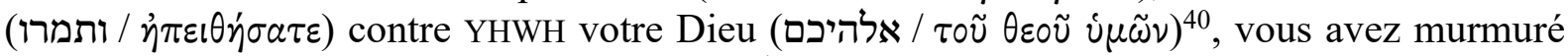

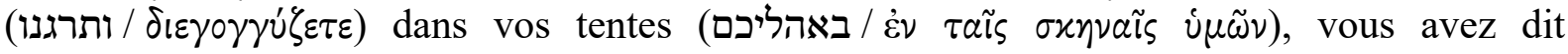

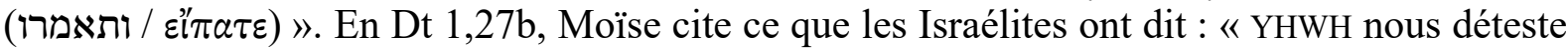

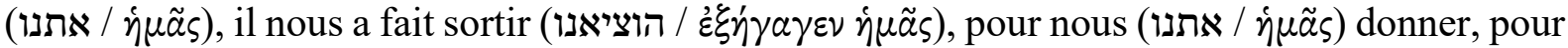

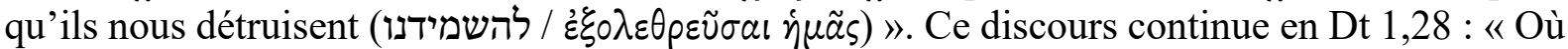

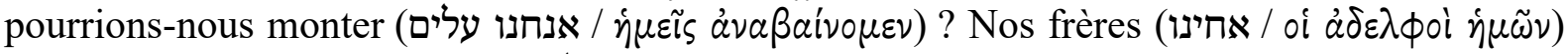
nous ont fait perdre courage (המסו את לבבנו / המש / les paroles de ceux qui ont découragé les Israélites sont citées, toujours à la $1^{\text {re }}$ personne du pluriel: «C'est un peuple plus grand que nous (ממנו / מ $/ \dot{\eta} \tilde{\omega} \nu)$, nous y avons vu (ראינו /

Après ces citations, Moïse continue à s'adresser aux Israélites en Dt 1,29-30, en reprenant

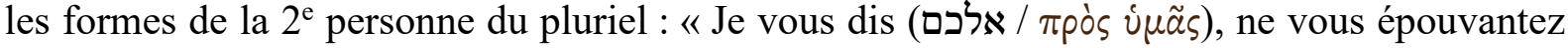

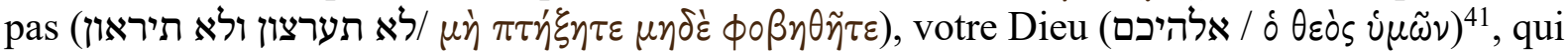

\footnotetext{
38 4QDeut ${ }^{\text {h }}$ suit le TM pour les verbes qui ont été conservés, comme le fait le SamP.

${ }^{39}$ Pour Dt 1,28, il faut remarquer la différence entre l'édition de RAHLFs 2006, p. 285 (oi ả $\delta \varepsilon \lambda \phi o i ~ u ́ \mu \omega \tilde{\nu}$

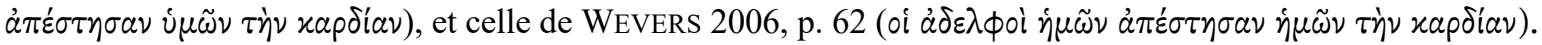

${ }^{40}$ Le Papyrus 963 présente une $1{ }^{\text {re }}$ personne du pluriel $(\dot{\eta} \mu \tilde{\omega} \nu)$, considérée par WeVERS 1978, p. 53 comme une erreur.

${ }^{41}$ Plusieurs manuscrits grecs, comme le Papyrus 963 (KenYon 1935, p. 37), ont une $1^{\text {re }}$ personne du pluriel $(\dot{\eta} \mu \tilde{\omega} \nu)$. Voir WeVERS 1978, p. 53.
} 


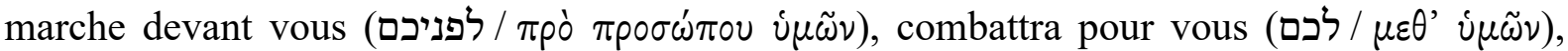

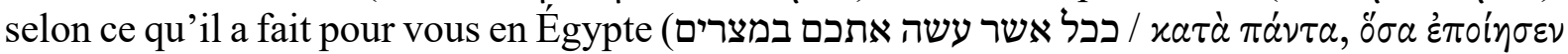

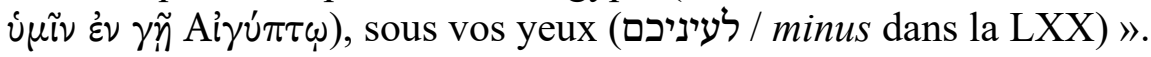

Ce n'est qu'en Dt 1,31 qu'on rencontre un nouveau « Numeruswechsel » dans le TM comme dans le SamP (il n'y a pas de témoins de cette partie du verset parmi les manuscrits de la Mer morte $)^{42}$. Ici, de manière imprévue, Moïse continue son discours aux Israélites à la $2^{\mathrm{e}}$ personne du singulier: « Tu as vu (ראית) que YHWH, ton Dieu (אלהיך), t'a porté (נשאך) 》. Dans la LXX,

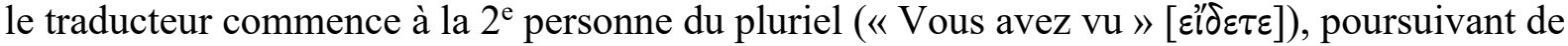
cette manière le pluriel des versets précédents ; cependant, pour les deux autres formes, il suit

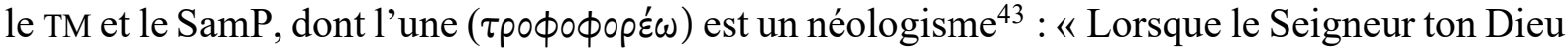

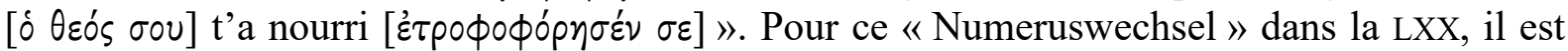
difficile de trouver une explication judicieuse ${ }^{44}$.

Dans la $2^{\mathrm{e}}$ partie du verset déjà, après ces quelques mots au singulier, l'auteur continue à la $2^{\mathrm{e}}$ personne du pluriel, dans le TM comme dans la LXX. C'est le cas jusqu'à la fin du chapitre, ici et là interrompu par quelques discours directs, dans lesquels Moïse cite les paroles que YHWH lui a adressées ${ }^{45}$.

Finalement, Dt 1,39 est un verset particulier. À part un minus dans la LXX (et dans 4QDeut ${ }^{\mathrm{h}}$; SamP suit la leçon du TM), il y a une autre variante remarquable. Le TM réfère aux "petits enfants qui ne connaissent ni le bien ni le mal» (וטפכם ... אשר לא ידעו היום טוב ורע). À cause du plus $\pi \tilde{\alpha} \nu$, le substantif $\pi a$ ı $\delta$ íov est au singulier, ce qui implique que le verbe suivant est

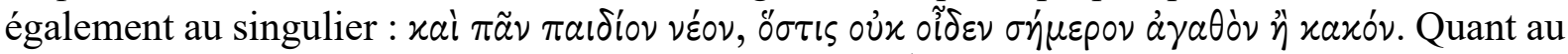

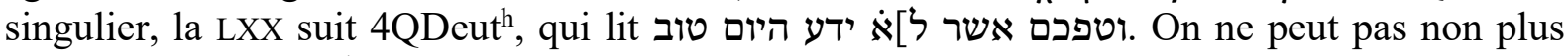
exclure que 4QDeut ${ }^{\mathrm{h}}$ ait également une variante dans la $1^{\text {re }}$ partie du verset. En tout cas, au moins en théorie, un équivalent hébreu (par exemple וכל טף אשר ל] correspondrait à xai $\pi \tilde{a} \nu \pi \alpha ı \delta$ íov $\nu \varepsilon ́ o v$ en 4QDeut ${ }^{\mathrm{h}}$ ne serait pas impossible ${ }^{46}$. Néanmoins, dans la LXX, la phrase relative suivante reprend de nouveau le nombre du TM : " ce sont eux qui y

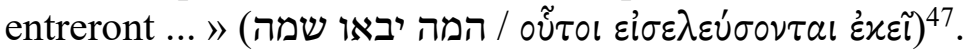

\section{Conclusion}

Déjà en Dt 1 , le phénomène du « Numeruswechsel » se présente de manière assez complexe, qui dépasse clairement la simple alternance entre la $2^{\mathrm{e}}$ personne du pluriel et la $2^{\mathrm{e}}$ personne du singulier dans le discours que Moïse adresse aux Israélites (le « Numeruswechsel » classique). Ainsi, si on compare le TM et la LXX de Dt 1, au moins trois catégories de « Numeruswechsel » peuvent être distinguées :

(1) Dans le TM, il y a des «Numeruswechsel» entre la $2^{\mathrm{e}}$ personne du singulier et la $2^{\mathrm{e}}$ personne du pluriel. En Dt 1, ces « Numeruswechsel » ne figurent plus dans la LXX. C'est le cas

${ }^{42}$ CAZelles 1967, p. 209, suggère une couche différente en Dt 1,31a.

${ }^{43}$ Voir Dogniez, Harl 2007, p. 118-119; Wevers 1995, p. 18 ; Den Hertog, Labahn, Pola 2011, p. 539.

${ }^{44}$ HemPel 1914, p. 8, considérait la version de la LXX comme une harmonisation par le traducteur.

${ }^{45}$ On ne peut que remarquer la $1^{\text {re }}$ personne pluriel $(\tau \omega \theta \varepsilon \omega \nu \mu \omega \omega \nu)$ dans le Papyrus 963 , au lieu de la $2^{\mathrm{e}}$ personne ( $\tau \tilde{\omega} \theta \varepsilon \tilde{\omega} \tilde{u} \dot{u} \mu \tilde{\omega} \nu$ ) dans les éditions de RAHLFS, HAHNHART 1935 et WEVERs 2006 en Dt 1,32. En Dt 1,35, dans le TM, YHWH réfère dans le discours de Moïse à la terre « qu'il a juré à vos ancêtres (לאבתיכם) 》. WeVERS 2006 suit cette

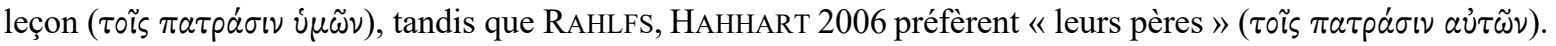

${ }^{46}$ Si c'était le cas, l'évaluation de WEVERS 1995, p. 23 n. 71, que la LXX offre « a free rendering » ne serait pas correcte.

${ }^{47}$ Pour ce verset, voir AUSLOOS 2001, p. 422-423; 427. 
en Dt 1,8 , où la forme à la $2^{\mathrm{e}}$ personne du singulier dans le discours de Moïse dans le TM est passée au pluriel dans la LXX (ce qui est également le cas en Dt 1,20). À cause du fait que 2QDeut ${ }^{\mathrm{a}}$ pour Dt 1,8 lit également une forme au pluriel, il est au moins plausible que, dans ce verset, le traducteur de la LXX se soit basé sur une Vorlage différente.

Un autre «Numeruswechsel» dans le TM se trouve en Dt 1,20.31. Dans ces versets également (pour Dt 1,31 de manière partielle), la LXX semble avoir harmonisé avec le contexte.

(2) En Dt 1,8 (MT), on rencontre un «Numeruswechsel» entre la $1^{\text {re }}$ et la $3^{\mathrm{e}}$ personne $d u$ singulier. De nouveau, dans la LXX, ce « Numeruswechsel » n'existe pas/plus, mais cette foisci en concordance avec la lecture du SamP. Le fait qu'une formule identique soit utilisée en Dt 1,35 rend plausible que le traducteur ou sa Vorlage aient harmonisé Dt 1,8 avec ce verset.

(3) Le «Numeruswechsel» en Dt 1,17 est assez particulier. Là, il y a un « Numeruswechsel » au niveau de la LXX, qui ne figure ni dans le TM, ni dans le SamP. Dans ce cas-ci, il faut se poser la question s'il serait imaginable que le traducteur (ou sa Vorlage) ait harmonisé avec le contexte plus large (Dt 16,19).

(4) Finalement, il faut noter que, dans plusieurs cas, les manuscrits d'une même tradition (TM ou LXX) ne sont pas toujours identiques. Dans ce contexte, les variantes entre le Papyrus 963 et les éditions de Rahlfs et du Göttingen Unternehmen, en ce qui concerne Dt 1,21.30, valent la peine d'être signalées.

Il n'est pas possible d'arriver à des conclusions définitives concernant la réception du « Numeruswechsel » du texte hébreu dans la traduction grecque à partir de l'analyse d'un seul chapitre. Néanmoins, et en tout cas, l'analyse présente a illustré que ce phénomène est beaucoup plus complexe que présumé jusqu'ici.

\section{BIBLIOGRAPHIE}

Ausloos, H., 1999 : "The Risks of Rash Textual Criticism Illustrated on the Basis of the Numeruswechsel in Exod 23:20-33 », Biblische Notizen 97, p. 5-12.

— 2001 : «LXX Num 14 :23 : Once More a 'Deuteronomist' at Work? », dans B.A. TAYLOR (éd.), $X$ Congress of the International Organization for Septuagint and Cognate Studies Oslo, 1998 (Society of Biblical Literature. Septuagint and Cognate Studies Series 51), Atlanta, p. 415-427.

- 2014: "LXX's Rendering of the Numeruswechsel in the Book of Deuteronomy. Deuteronomy 12 as a Test Case », dans J.M. RoBKer, F. UEBERSCHAER, T. WAGNER (éd.), Text-Textgeschichte - Textwirkung. Festschrift zum 65. Geburtstag von Siegfried Kreuzer (Alter Orient und Altes Testament 419), Münster, p. 303-313.

- 2018: «One to three... Some Aspects of the Numeruswechsel within the LXX of Deuteronomy », dans M. Meiser, M. Geiger, S. Kreuzer, M. Sigismund (éds), Die Septuaginta - Geschichte, Wirkung, Relevanz (Wissenschaftliche Untersuchungen zum Neuen Testament 405), Tübingen, p. 202-214.

- 2022 : «How the Septuagint Can Shed New Light on the Dead Sea Scrolls. The Case of Deuteronomy 1,8 in 2QDeut ${ }^{\mathrm{a}}{ }^{\prime}$, dans W. KRAUS, M. KARRER, S. KREUZER, J. PersCh (éd.), Bibel und Patristik. Festschrift für Prof. Dr. Martin Meiser (Biblische Zeitschrift Supplements 3), Leiden, Boston, Singapore, Paderborn, p. 49-59. 
BEGG, C., 1978 : Contributions to the Elucidation of the Composition of Deuteronomy with Special Attention to the Significance of the Numeruswechsel (Doctoral dissertation Faculty of Theology KU Leuven), Leuven.

— 1979 : «The Significance of the Numeruswechsel in Deuteronomy. The 'Pre-History' of the Question », Ephemerides Theologicae Lovanienses 55, p. 116-124.

— 1994 : « 1994 : A Significant Anniversary in the History of Deuteronomy Research », dans F. GARCÍA MARTínEZ et al. (éd.), Studies in Deuteronomy. FS C.J. Labuschagne (Supplements to Vetus Testamentum 53), Leiden, New York, Köln, p. 1-11.

Bertholet, A., 1899 : Deuteronomium (Kurzer Hand-Commentar zum Alten Testament 5), Freiburg im Breisgau, Leipzig, Tübingen.

CAzelles, H., 1967 : «Passages in the Singular within Discourse in the Plural of Dtn 1-4 », Catholic Biblical Quarterly 29, p. 207-219.

Den Hertog, C., Labahn, M., Pola, T., 2011 : « Deuteronomion / Deuteronomium / Das fünfte Buch Mose », dans M. Karrer, W. Kraus (éd.), Septuaginta Deutsch. Erläuterungen und Kommentare zum griechischen Alten Testament. Band I : Genesis bis Makkabäer, Stuttgart, p. 523-601.

Dogniez, C., Harl, M., 2007 : Le Deutéronome. Traduction du texte grec de la Septante, introduction et notes (La Bible d'Alexandrie 5), Paris.

HemPel, J., 1914 : Die Schichten des Deuteronomium. Ein Beitrag zur israelitischen Literaturund Rechtsgeschichte, Leipzig.

HOSPERS, J.H., 1947 : De Numeruswisseling in het boek Deuteronomium, Utrecht.

JoÜON, P, MuraOKA, T, 1993 : A Grammar of Biblical Hebrew (Subsidia Biblica 14), Rome.

Kenyon, F.G., 1935 : The Chester Beatty Biblical Papyri. Descriptions and Texts of Twelve Manuscripts on Papyrus of the Greek Bible. Fasciculus V: Numbers and Deuteronomy, London, 1935.

KÖNIG, E., 1917 : Das Deuteronomium eingeleitet, übersetzt und erklärt (Kommentar zum Alten Testament 3), Leipzig.

LABERge, L., 1985 : « La Septante de Dt 1-11», dans N. Lohfink (éd.), Das Deuteronomium. Entstehung, Gestalt und Botschaft (Bibliotheca Ephemeridum Theologicarum Lovaniensium 68), Leuven, p. 129-134.

LOHFINK, N., 1963 : Das Hauptgebot. Eine Untersuchung literarischer Einleitungsfragen zu Dtn 1-11 (Analecta Biblica 20), Rome.

— 2004 : « Deuteronomium 1,9-18 : Gerichtsverfassung und Militär », dans E. Bons (éd.), Le jugement dans l'un et l'autre testament. Mélanges offerts à Raymond Kuntzmann (Lectio Divina 197), Paris, p. 81-108.

McCARTHY, C., 2007 : אלה הדברים-Deuteronomy (Biblia Hebraica Quinta 5), Stuttgart.

Oтто, E., 2012 : Deuteronomium 1-11. Erster Teilband: 1,1-4,43 (Herders Theologischer Kommentar zum Alten Testament), Freiburg, Basel, Wien.

PUUKO, A.F., 1910 : Das Deuteronomium. Eine literarkritische Untersuchung, Leipzig.

RAHLFS, A., HANHART, R., 2006 : Septuaginta id est Vetus Testamentum graece iuxta LXX interpres. Edition altera, Stuttgart. 
RÜTERSWÖRDEN, U., 2006 : Das Buch Deuteronomium (Neuer Stuttgarter Kommentar Altes Testament 4), Stuttgart.

SMITH, G.A., 1918 : The Book of Deuteronomy (Cambridge Bible for Schools and Colleges), Cambridge.

StAerk, W., 1894 : Das Deuteronomium. Sein Inhalt und seine literarische Form. Eine kritische Studie, Leipzig.

Steuernagel, C., 1894 : Der Rahmen des Deuteronomiums. Literarcritische Untersuchungen über seine Zusammensetzung und Entstehung, Halle an der Saale.

- 1898 : Das Deuteronomium übersetzt und erklärt (Handkommentar zum Alten Testament 1/3), Göttingen.

Tal, A., Florentin, M. 2010 : The Pentateuch. The Samaritan Version and the Masoretic Version, Tel Aviv.

Ulrich, E., 2010: The Biblical Qumran Scrolls. Transcriptions and Textual Variants (Supplements to Vetus Testamentum 134), Leiden, Boston.

Veijola, T., 1988 : «Principal Observations on the Basic Story in Deuteronomy 1-3 », dans M. Augustin, K.-D. SchuncK (éd.), "Wünschet Jerusalem Frieden”. Collected Communications to the XIIth Congress of the International Organization for the Study of the Old Testament. Jerusalem 1986, Frankfurt, p. 249-259.

WeVers, J.W., 1978 : Text History of the Greek Deuteronomy (Mitteilungen des SeptuagintaUnternehmens 13), Göttingen, 1978.

- 1995 : Notes on the Greek Text of Deuteronomy (Society of Biblical Literature. Septuagint and Cognate Studies 39), Atlanta.

- 2006 : Deuteronomium (Septuaginta Vetus Testamentum Graecum Auctoritate Academiae Scientiarum Gottingensis editum 3/2), Göttingen, ${ }^{2} 2006$. 


\title{
RÉSUMÉ
}

L'échange entre la $2^{\mathrm{e}}$ personne du singulier et la $2^{\mathrm{e}}$ personne du pluriel dans le discours d'adieu de Moïse adressé aux Israélites compte sans doute parmi les caractéristiques les plus énigmatiques du livre du Deutéronome. Cette variation, qui est connue sous le terme technique du « Numeruswechsel » n'a pas encore été étudiée de manière systématique au niveau de la « réception » dans la Septante (LXX).

Dans cette contribution, une analyse est faite de tous les cas du « Numeruswechsel» dans les témoins textuels hébreux (Texte massorétique, Qumran et Pentateuque samaritain) et grecs (LXX) du $1^{\text {er }}$ chapitre du livre du Deutéronome. Elle révèle surtout la complexité du problème, en montrant que le «Numeruswechsel » dépasse la seule variation entre la $2^{\mathrm{e}}$ personne du pluriel et du singulier dans le discours de Moïse aux Israélites.

\begin{abstract}
Undoubtedly, the exchange between the $2^{\text {nd }}$ person singular and the $2^{\text {nd }}$ person plural in Moses' farewell address to the Israelites is one of the most enigmatic features of the book of Deuteronomy. This variation, which is known under the technical term 'Numeruswechsel', has not yet been systematically studied at the level of its 'reception' in the Septuagint (LXX).

In this contribution, an analysis is made of all the cases of the 'Numeruswechsel' in the Hebrew (Massoretic Text, Qumran and Samaritan Pentateuch) and Greek (LXX) textual witnesses of the $1^{\text {st }}$ chapter of the book of Deuteronomy. Above all, it points out the complexity of the problem, demonstrating that the problem of the 'Numeruswechsel' largely surpasses the variation between $2^{\text {nd }}$ person plural and singular in Moses' speech to the Israelites.
\end{abstract}

\section{MOTS-CLEFS}

1. Deutéronome

2. Numeruswechsel

3. Septante

4. Critique textuelle

5. Texte massorétique

\section{KEYWORDS}

1. Deuteronomy

2. Numeruswechsel

3. Septuagint

4. Textual Criticism

5. Masoretic text 